\title{
Nicotinamide promotes pancreatic differentiation through the dual inhibition of CK1 and ROCK kinases in human embryonic stem cells
}

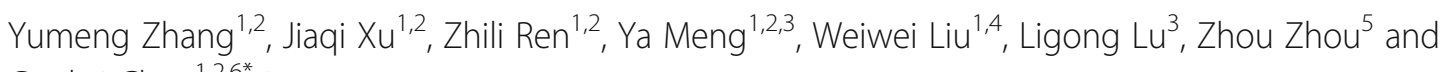
Guokai Chen ${ }^{1,2,6^{*}}$ (i)

\begin{abstract}
Background: Vitamin B3 (nicotinamide) plays important roles in metabolism as well as in SIRT and PARP pathways. It is also recently reported as a novel kinase inhibitor with multiple targets. Nicotinamide promotes pancreatic cell differentiation from human embryonic stem cells (hESCs). However, its molecular mechanism is still unclear. In order to understand the molecular mechanism involved in pancreatic cell fate determination, we analyzed the downstream pathways of nicotinamide in the derivation of NKX6.1 $1^{+}$pancreatic progenitors from hESCs.

Methods: We applied downstream modulators of nicotinamide during the induction from posterior foregut to pancreatic progenitors, including niacin, PARP inhibitor, SIRT inhibitor, CK1 inhibitor and ROCK inhibitor. The impact of those treatments was evaluated by quantitative real-time PCR, flow cytometry and immunostaining of pancreatic markers. Furthermore, CK1 isoforms were knocked down to validate CK1 function in the induction of pancreatic progenitors. Finally, RNA-seq was used to demonstrate pancreatic induction on the transcriptomic level.

Results: First, we demonstrated that nicotinamide promoted pancreatic progenitor differentiation in chemically defined conditions, but it did not act through either niacin-associated metabolism or the inhibition of PARP and SIRT pathways. In contrast, nicotinamide modulated differentiation through CK1 and ROCK inhibition. We demonstrated that CK1 inhibitors promoted the generation of PDX1/NKX6.1 double-positive pancreatic progenitor cells. shRNA knockdown revealed that the inhibition of CK1a and CK1 $\varepsilon$ promoted pancreatic progenitor differentiation. We then showed that nicotinamide also improved pancreatic progenitor differentiation through ROCK inhibition. Finally, RNA-seq data showed that CK1 and ROCK inhibition led to pancreatic gene expression, similar to nicotinamide treatment.
\end{abstract}

\footnotetext{
* Correspondence: guokaichen@um.edu.mo

${ }^{1}$ Centre of Reproduction, Development and Aging, Faculty of Health

Sciences, University of Macau, Macau SAR, China

${ }^{2}$ Institute of Translational Medicine, Faculty of Health Sciences, University of Macau, Macau SAR, China

Full list of author information is available at the end of the article
}

(C) The Author(s). 2021 Open Access This article is licensed under a Creative Commons Attribution 4.0 International License, which permits use, sharing, adaptation, distribution and reproduction in any medium or format, as long as you give appropriate credit to the original author(s) and the source, provide a link to the Creative Commons licence, and indicate if changes were made. The images or other third party material in this article are included in the article's Creative Commons licence, unless indicated otherwise in a credit line to the material. If material is not included in the article's Creative Commons licence and your intended use is not permitted by statutory regulation or exceeds the permitted use, you will need to obtain permission directly from the copyright holder. To view a copy of this licence, visit http://creativecommons.org/licenses/by/4.0/ The Creative Commons Public Domain Dedication waiver (http://creativecommons.org/publicdomain/zero/1.0/) applies to the data made available in this article, unless otherwise stated in a credit line to the data. 
Conclusions: In this report, we revealed that nicotinamide promotes generation of pancreatic progenitors from hESCs through CK1 and ROCK inhibition. Furthermore, we discovered the novel role of CK1 in pancreatic cell fate determination.

Keywords: Human embryonic stem cells, Pancreatic progenitors, Nicotinamide, Casein kinase 1 (CK1), Rhoassociated protein kinase (ROCK), Kinase inhibitor

\section{Background}

Unexpected side effects of a specific drug often imply additional targets. These off-target phenomena are valuable resources to reveal novel molecular mechanisms and could help to identify new applications of clinically approved drugs. Human embryonic stem cells (hESCs) are pluripotent and can be differentiated into all cell types. hESC differentiation provides an excellent platform for people to examine key biological processes [14]. By delineating the molecular mechanisms of offtarget effects in stem cell differentiation, new stem cell applications could be developed from known modulators.

Nicotinamide (NAM) is a multi-target drug that is widely used as a topical treatment for acne, eczema and other skin conditions. Nicotinamide belongs to the vitamin $B_{3}$ family that also includes niacin. Vitamin $B_{3}$ are converted to the coenzyme nicotinamide adenine dinucleotide (NAD) that is essential for energy metabolism [5]. Micromolar-range nicotinamide is sufficient to carry out metabolic functions. When its concentration is elevated to millimolar level, nicotinamide becomes a multitarget compound that is widely utilized in disease treatments and cell culture [6]. Nicotinamide can regulate DNA repair and apoptosis by inhibiting $\mathrm{NAD}^{+}$dependent enzymes such as poly (ADP-ribose) polymerases (PARP) [7]. Nicotinamide can also inhibit Sirtuins (SIRT) to influence epigenetic modification and metabolism [8]. Because of the complexity of kinase cascades and their crosstalk with other pathways, much is unknown about nicotinamide's function as a kinase inhibitor in disease treatments and cell culture.

Nicotinamide has been widely applied in various aspects of stem cell culture for hESC and human-induced pluripotent stem cells (hiPSC). Nicotinamide suppresses apoptosis and improves reprogramming efficiency [9, 10]. Nicotinamide is also beneficial for the differentiation toward different somatic cell types, such as $\mathrm{CD} 34^{+}$ hematopoietic progenitors [11], retinal pigment epithelium (RPE) [12], and cardiomyocytes from hESC or hiPSC [13]. hESCs have been induced to pancreatic progenitors, putative $\beta$ cells, or bona fide $\beta$ cells by nicotinamide [14-19]. Nicotinamide promotes the development and self-renewal of murine pancreatic progenitors [20], and it also sustains the expression of panpancreas marker PDX1 [21] and endocrine marker
NKX6.1 in the development of human pancreatic progenitors in serum-containing conditions [22]. However, the mechanism of nicotinamide in pancreatic lineage differentiation remains unclear. Although PARP and SIRT pathways are involved in nicotinamide-associated differentiation, little data is available to validate the mechanism of nicotinamide in cell fate determination processes. We recently show that nicotinamide promotes cell survival as a ROCK inhibitor and induces RPE through CK1 inhibition [6].

Nicotinamide is beneficial for pancreatic progenitor induction, but its downstream effector is unclear in this process. In order to understand the mechanism of nicotinamide in pancreatic in vitro development, we established a serum-free, chemically defined platform to analyze its function in pancreatic differentiation. We hope to reveal critical signaling pathways in pancreatic progenitor differentiation and develop novel methods to generate pancreatic cells from human pluripotent stem cells.

\section{Materials and methods hESC culture}

Human ESCs (H1 and H9 lines from WiCell Research Institute, Inc., Madison, WI, http://www.wicell.org) were cultured in Matrigel-coated 6-well plates in E8 medium $[23,24]$. Medium was changed daily, and cells were passaged every 3-4days before they reach $60-70 \%$ confluence.

\section{Pancreatic progenitor differentiation}

The differentiation was carried out in serum-free conditions as illustrated in Fig. 1a. Sixty to $70 \%$ confluent hESCs were dissociated with EDTA, passaged onto Matrigel-coated 24-well plate (passaging ratio 1:24), and cultured until $40-50 \%$ confluence. Cells were then treated with $5 \mu \mathrm{M}$ CHIR99021 and $100 \mathrm{ng} / \mathrm{ml}$ Activin A in differentiation medium (DMEM/F12, transferrin, chemically defined lipid concentrate, ascorbic acid, and sodium selenite) for $24 \mathrm{~h}$, followed by $100 \mathrm{ng} / \mathrm{ml}$ Activin A, $100 \mathrm{nM}$ LDN193189, and $2 \mu \mathrm{M}$ IWP2 treatment in differentiation medium for another $48 \mathrm{~h}$ to induce definitive endoderm formation (Stage 1). Subsequently, primitive gut tube cells were induced under $50 \mathrm{ng} / \mathrm{ml}$ KGF in DMEM/F12 with $0.2 \% \mathrm{NaHCO}_{3}$ and $1 \times$ GlutaMAX (Stage 2). Posterior foregut cells were induced 

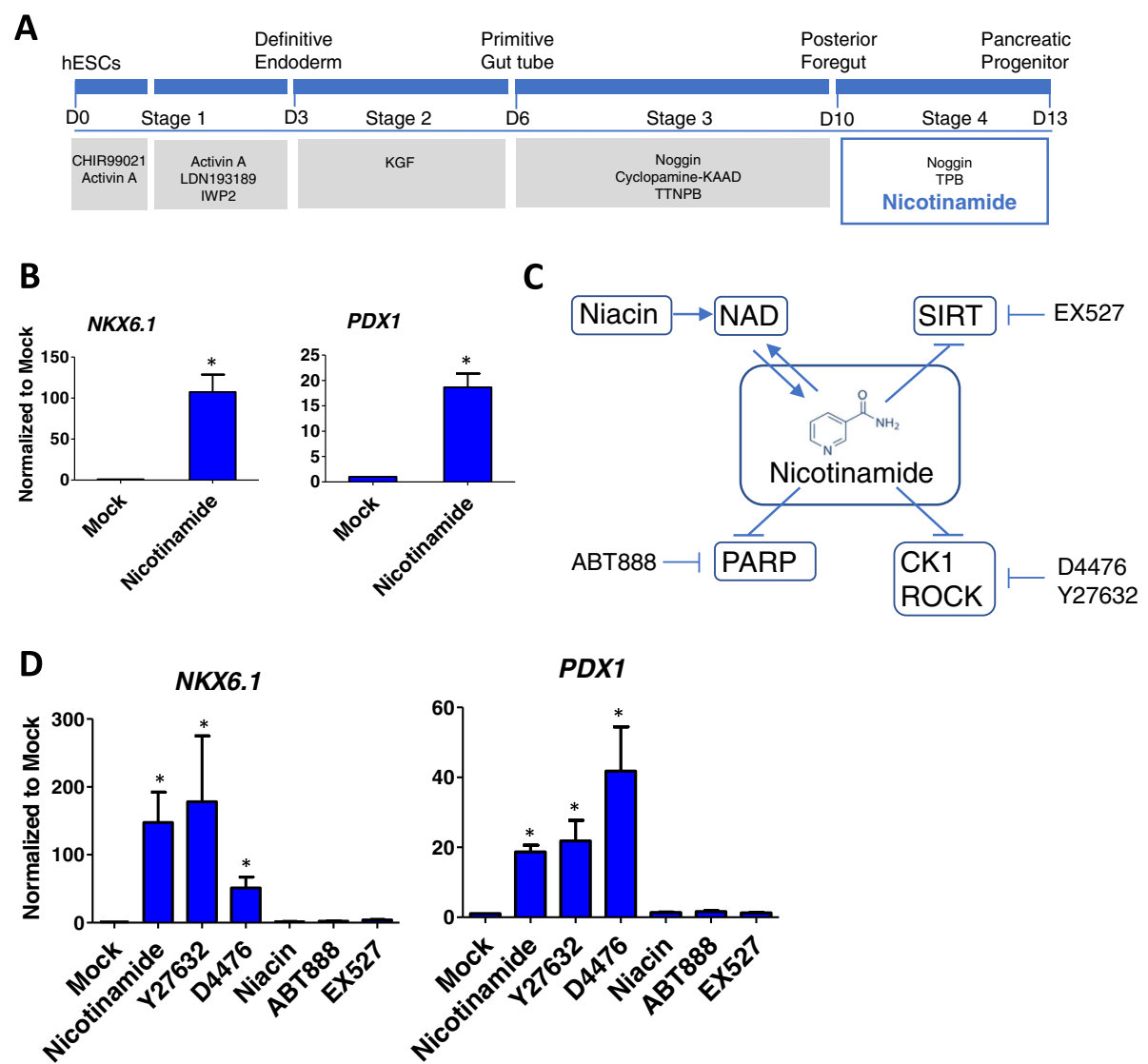

Fig. 1 Enhancement of pancreatic progenitor induction by nicotinamide depends on kinase inhibition, but not on PARP and SIRT regulation. a Four-stage differentiation strategy to induce pancreatic progenitors from hESCs; b RT-qPCR analysis for mRNA levels of NKX6.1 and PDX1 in cells on day 13 of differentiation, treated with nicotinamide $(n=3),{ }^{*} p<0.05$; c Nicotinamide inhibits PARP, SIRT and several kinases, and contributes to NAD synthesis; d RT-qPCR analysis for mRNA level of NKX6.1 and PDX1 in cells on day 13 treated with nicotinamide $(10 \mathrm{mM})$, Y27632 (10 $\mu \mathrm{M})$, D4476 $(5 \mu \mathrm{M})$, niacin $(5 \mathrm{mM})$, PARP inhibitor ABT888 (50 nM) and SIRT inhibitor EX527 $(10 \mu \mathrm{M})(\mathrm{n}=3),{ }^{*} p<0.05$

under $100 \mathrm{ng} / \mathrm{ml}$ Noggin, $1 \mathrm{mM}$ cyclopamine-KAAD, $10 \mu \mathrm{M}$ TTNPB treatment in DMEM HG with $1 \%$ B27 (without insulin), and 1× GlutaMAX (Stage 3). Subsequently, pancreatic progenitors were generated under $100 \mathrm{ng} / \mathrm{ml}$ Noggin and $500 \mathrm{nM}$ TPB treatment in DMEM HG with 1\% B27 (without insulin), 1× GlutaMAX, and factors involved in nicotinamide-associated pathways as specified (Stage 4). Chemicals used include nicotinamide $(10 \mathrm{mM})$, niacin $(1,5,10 \mathrm{mM})$, PARP inhibitor ABT888 (10, 50, $500 \mathrm{nM})$, SIRT inhibitor EX527 $(1,10,20 \mu \mathrm{M})$, ROCK inhibitor Y27632 $(10 \mu \mathrm{M})$, CK1 inhibitor D4476 $(5 \mu \mathrm{M})$, PF4800567 $(10 \mu \mathrm{M})$ and LH846 $(10 \mu \mathrm{M})$.

\section{Real-time PCR}

Total mRNA was extracted with RNAiso-plus (TAKA RA, cat. no.108-95-2), and reverse transcription from mRNA to cDNA was performed with High Capacity cDNA Reverse Transcription kit (Applied Biosystems, cat. no. 4368813) following the manufacturer's instructions. Real-time quantitative PCR was conducted with SYBR Premix Ex Taq (TAKARA, cat. no. RR420) and the Quantstudio-7 system (Applied Biosystems). The relative amounts of the amplified nucleotide fragment were calculated by the $2^{\wedge}(-\Delta \mathrm{Ct})$ method. Expression levels were normalized to the housekeeping gene TBP and compared with undifferentiated hESCs.

\section{Immunostaining}

Putative pancreatic progenitors were fixed with $4 \%$ paraformaldehyde at room temperature for $20 \mathrm{~min}$, rinsed with $1 \times$ PBS 3 times, permeabilized with $0.5 \%$ Triton X100 for $20 \mathrm{~min}$, and then stained with primary and secondary antibodies following standard protocols. Primary antibodies include goat anti-PDX1 (Cell Signaling, cat. no. 5679 at 1:2000 dilution) and mouse anti-NKX6.1 (BD biosciences, cat. no. 563338 at 1:200 dilution). The nuclei were stained with Hoechst 33342 (Abnova, cat. no. U0334 at 1:10,000 dilution). Stained cells were 
visualized using Zeiss Axio Observer fluorescence microscope with ApoTome.

\section{Flow cytometry}

Putative pancreatic progenitors were harvested by TrypLE $\left(37^{\circ} \mathrm{C}, 5 \mathrm{~min}\right)$ and neutralized with $5 \% \mathrm{FBS}$. After washing with DPBS, cells were permeabilized with $0.2 \%$ Triton X-100, 5\% FBS in DPBS for $1 \mathrm{~h}$ on ice. Primary antibodies goat anti-human PDX1 (Cell Signaling, cat. no. 5679 at 1:1000 dilution) and mouse anti-human NKX6.1 conjugated by Alexa Fluor 647 (BD Biosciences, cat. no. 563338 at 1:20 dilution) were incubated with cells in $1 \%$ BSA in DPBS for $1 \mathrm{~h}$ at room temperature in the dark. After washing, Alexa Fluor ${ }^{\circ}$ 488-conjugated anti-goat secondary antibody was used at 1:1000 dilution in 1\% BSA in DPBS for $1 \mathrm{~h}$ at room temperature in the dark for PDX1 staining. After washing, cells were resuspended in DPBS for analysis using BD ACCURI C6. Undifferentiated hESCs were stained with PDX1 or NKX6.1 as a negative control for gating.

\section{Western blot}

Cells were lysed with RIPA buffer supplemented with phosphatase inhibitor cocktail (Sigma-Aldrich, cat. No. P5726) and proteinase inhibitors. The lysate was sonicated and heated at $95^{\circ} \mathrm{C}$ before loading onto $12 \%$ sodium dodecyl sulfate-polyacrylamide gel (SDS-PAGE), and the separated proteins were transferred to PVDF membrane. The membrane was blocked with 5\% BSA in TBST at room temperature for $30 \mathrm{~min}$, washed with TBST ( 5 min each time, 3 times), incubated with primary antibodies at $4{ }^{\circ} \mathrm{C}$ overnight, washed with TBST (5 min each time, 3 times), and incubated with peroxidaseconjugated secondary antibodies. Signal strength was semi-quantitatively determined by optical densitometry using ImageJ Lab. Primary antibodies include phospho$\beta$-catenin (Ser45) (Cell Signaling, cat. no. $9561 \mathrm{~s}$ at 1 : 1000 dilution), $\beta$-catenin (Upstate, cat. no. 06-734 at 1 : 1000 dilution), phospho-MLC (Ser19) (Cell Signaling, cat. no. $3675 \mathrm{~s}$ at 1:2000 dilution), MLC (Sigma-Aldrich, cat. no. SAB1403431 at 1:2000 dilution) and GAPDH (Santa Cruz, cat. no. sc-25778 at 1:2000 dilution).

\section{Cloning and generation of shRNA in hESCs lines}

The psi-LVRU6P plasmid was utilized to create shRNAexpressing constructs. Target sites of shCK $1 \alpha$, shCK $1 \delta$ and shCK1s are GGAAGTGGCAGTGAAGCTAGA, GGTCCTTCGGAGATATCTACC and GGTTGCCATC AAGCTTGAATG. The lentivirus particles were produced in 293FT cells transfected with psPAX2, pMD2.G and psi-LVRU6P-shRNA-containing plasmids. H1 cells were transduced with the lentivirus and stable cell lines were established by puromycin selection.

\section{RNA sequencing}

Total mRNA was extracted on day 13 (stage 4) using RNAiso-plus (TAKARA, cat. no.108-95-2). The RNA libraries were generated using TruSeq RNA Sample Preparation kit (Illumina), and cDNA fragments were enriched by PCR using Illumina TruSeq PCR primers. Each library was sequenced as paired-end reads in HiSeq 2000/1000 (Illumina). The sequencing data of this report have been uploaded in NCBI's BioProject and Sequence Read Archive (SRA), and these data are accessible through BioProject accession number PRJNA701836 or SRA accession number SRP306377.

\section{Bioinformatics analysis}

Transcripts Per Million (TPM) were used to normalize gene read counts in all samples. Values for the heat map were calculated by $\log 2$ (TPM of each gene in each sam$\mathrm{ple} /$ mean TPM of each gene in all samples). $\mathrm{R}$ package gplots was used to generate heatmaps. $R$ package Edge $R$ was used to pick differentially expressed genes (DEG) with $\mathrm{p}$ value $<0.01, \log 2$ (fold change) $>2$ or $<-2$. The Euclidean distances between selected genes in each sample were used as the clustering method for the heatmap cluster. Cell type enrichment analysis was performed using the section of "GTEx Tissue Sample Gene Expression Profiles up" in Enrichr (https://maayanlab.cloud/ Enrichr/).

\section{Statistical analysis}

Data represent mean \pm SEM of $\geq 3$ independent experiments unless specified. Statistical significance was tested by either paired $\mathrm{t}$ test or one-way ANOVA analysis. $p<$ 0.05 represents statistical significance.

\section{Result}

Enhancement of pancreatic progenitor induction by nicotinamide depends on kinase inhibition, but not on PARP and SIRT regulation

Nicotinamide has been used to induce pancreatic progenitors from hESCs [21, 22], but its molecular mechanism is unclear. In order to examine the function of nicotinamide, a serum-free platform was established to stepwisely induce pancreatic progenitors (Fig. 1a). After H1 hESCs differentiated to posterior foregut (Stage 3), noggin and TPB were applied to induce pancreatic progenitors (Stage 4). We showed that the addition of nicotinamide in stage 4 significantly enhanced the expression of pancreatic progenitor markers NKX6.1 and PDX1 (Fig. 1b). This system was utilized to study nicotinamide regulation of pancreatic specification in this report.

Nicotinamide is best known to serve as the substrate to produce NAD as well as an inhibitor for PARP and SIRT pathways (Fig. 1c). Niacin was tested in Stage 4, but it failed to enhance pancreatic differentiation as 
shown by the mRNA levels of PDX1 and NKX6.1 and percentage of $\mathrm{PDX1}^{+} / \mathrm{NKX} 6.1^{+}$cells (Fig. 1d, Fig. S1c, d). It suggested that nicotinamide did not modulate differentiation through NAD metabolism. When PARP inhibitor (ABT888) and SIRT inhibitor (EX527) were applied during Stage 4, they did not improve NKX6.1 and PDX1 mRNA levels and percentage of $\mathrm{PDX}^{+} /$ NKX6.1 $1^{+}$cells (Fig. 1d, Fig. S1c, d). It indicated that nicotinamide did not modulate pancreatic differentiation through PARP and SIRT inhibition.

We recently found that nicotinamide was also a kinase inhibitor with multiple targets including ROCK and CK1 (Fig. S1a) [6], so we examined whether nicotinamide modulates pancreatic differentiation as a kinase inhibitor. When CK1 inhibitor D4476 and ROCK inhibitor Y27632 were individually applied in stage 4, each of them significantly increased the expression of $P D X 1$ and NKX6.1, and this phenotype was similar to that of nicotinamide (Fig. 1d, Fig. S1e). Besides the $\mathrm{H} 1$ cell line, we showed that H9 hESCs can also be induced to pancreatic progenitors as shown by the significant increase of the mRNA levels of PDX1 and NKX6.1 upon CK1 or ROCK inhibition (Fig. S1b). These results indicated that nicotinamide influenced pancreatic differentiation as a kinase inhibitor. The cell fate determination could be carried out through CK1 and ROCK in parallel.

\section{Generic chemical inhibition of CK1 promotes pancreatic progenitor induction}

We further examined how nicotinamide and CK1 affected pancreatic progenitor differentiation in Stage 4 (Fig. 1a). Similar to nicotinamide, CK1 inhibitor D4476 not only significantly increased the expression of NKX6.1 and PDX1, but also promoted the expression of additional pancreatic progenitor marker genes PTF1A and SOX9 as well as endocrine precursor marker NGN3 (Fig. 2a, Fig. S2a). Immunostaining results demonstrated that nicotinamide and D4476 both increased the expression of PDX1 and NKX6.1 (Fig. 2b), which were consistent with flow cytometry results (Fig. 2c). These results supported our hypothesis that nicotinamide induced pancreatic progenitors as a CK1 inhibitor.

We then inspected whether downstream factors of CK1 were responsible for the cell fate induction. Previous study revealed that GSK-3 dependent phosphorylation of $\beta$-catenin relies on prior priming phosphorylation of $\beta$-catenin at Ser45 by CK1 in canonical Wnt pathway [25]. We showed that both nicotinamide and D4476 suppressed the phosphorylation of $\beta$ Catenin (Ser45) in putative pancreatic progenitors (Fig. 2d, d'). This result indicated that nicotinamide and D4476 might promote pancreatic progenitor induction through Wnt pathway.

\section{Inhibition of CK1a and CK1 $1 \varepsilon$ promotes pancreatic progenitor induction}

The human genome contains multiple CK1 isoforms with distinct functions. D4476 is a pan CK1 inhibitor that targets $\mathrm{CK} 1 \alpha, \mathrm{CK} 1 \delta$ and CK1 1 . We tried to determine which CK1 isoform was involved in pancreatic differentiation. CK1 isoform-specific inhibitors were applied in Stage 4 (Fig. 1a), and we showed that CK1e inhibitor PF4800567 hydrochloride significantly improved the expression of pancreatic progenitor-specific marker genes as well as endocrine precursor marker NGN3 (Fig. 3a), and it also increased the percentage of $\mathrm{PDX}^{+} / \mathrm{NKX} 6.1^{+}$cells (Fig. 3b). In contrast, CK1 $\delta$ inhibitor LH 846 did not significantly improve pancreatic differentiation.

We then used shRNA to knockdown the gene expression of CK $1 \alpha$, CK $1 \delta$ and CK $1 \varepsilon$, respectively (Fig. S3a, b). The knockdown of CK $1 \alpha$ and CK1 $\varepsilon$ significantly upregulated the mRNA levels of typical pancreatic progenitor markers (NKX6.1, PDX1, PTF1A and SOX9) (Fig. 3c),

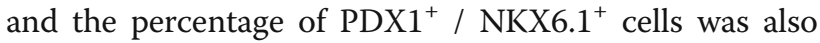
increased in these two knockdown cell lines (Fig. 3d, Fig. S3c). In contrast, CK1 $\delta$ knockdown did not improve pancreatic differentiation (Fig. 3c, d, Fig. S3c). These data indicated that CK1 $\alpha$ and CK1 $\varepsilon$ suppressed pancreatic differentiation, which was enhanced by CK1 inhibition.

\section{Nicotinamide also promotes pancreatic differentiation through ROCK inhibition}

Consistent with previous report on the targets of nicotinamide [6], we found that both CK1 and ROCK were involved in pancreatic progenitor differentiation under nicotinamide treatment (Fig. 1c). We compared the impact of nicotinamide and ROCK inhibitor Y27632 in Stage 4 of pancreatic differentiation. Both nicotinamide and Y27632 significantly improved the expression of pancreatic progenitor genes, including NKX6.1, PDX1, PTF1A and SOX9 (Fig. 4a), while NGN3 was only significantly upregulated under nicotinamide treatment. Nicotinamide or Y27632 had no significant impact on the mRNA level of endocrine marker NEUROD1 (Fig. S4a). Immunostaining showed that the expression of PDX1 and NKX6.1 was upregulated by nicotinamide and Y27632 treatments (Fig. 4b). Flow cytometry results also showed that both nicotinamide and Y27632 upregulated the percentage of $\mathrm{PDX} 1^{+} / \mathrm{NKX} 6.1^{+}$double-positive cells (Fig. 4c).

Myosin Light Chain 2 (MLC2) phosphorylation is downstream of ROCK kinase [26], so we evaluated MLC2 phosphorylation at Ser19 during Stage 4 (from day 10 to day 13). Under Noggin/TPB treatment, MLC2 phosphorylation gradually increased from day 10 to day 13 (Fig. 4d, d'). Both nicotinamide and Y27632 


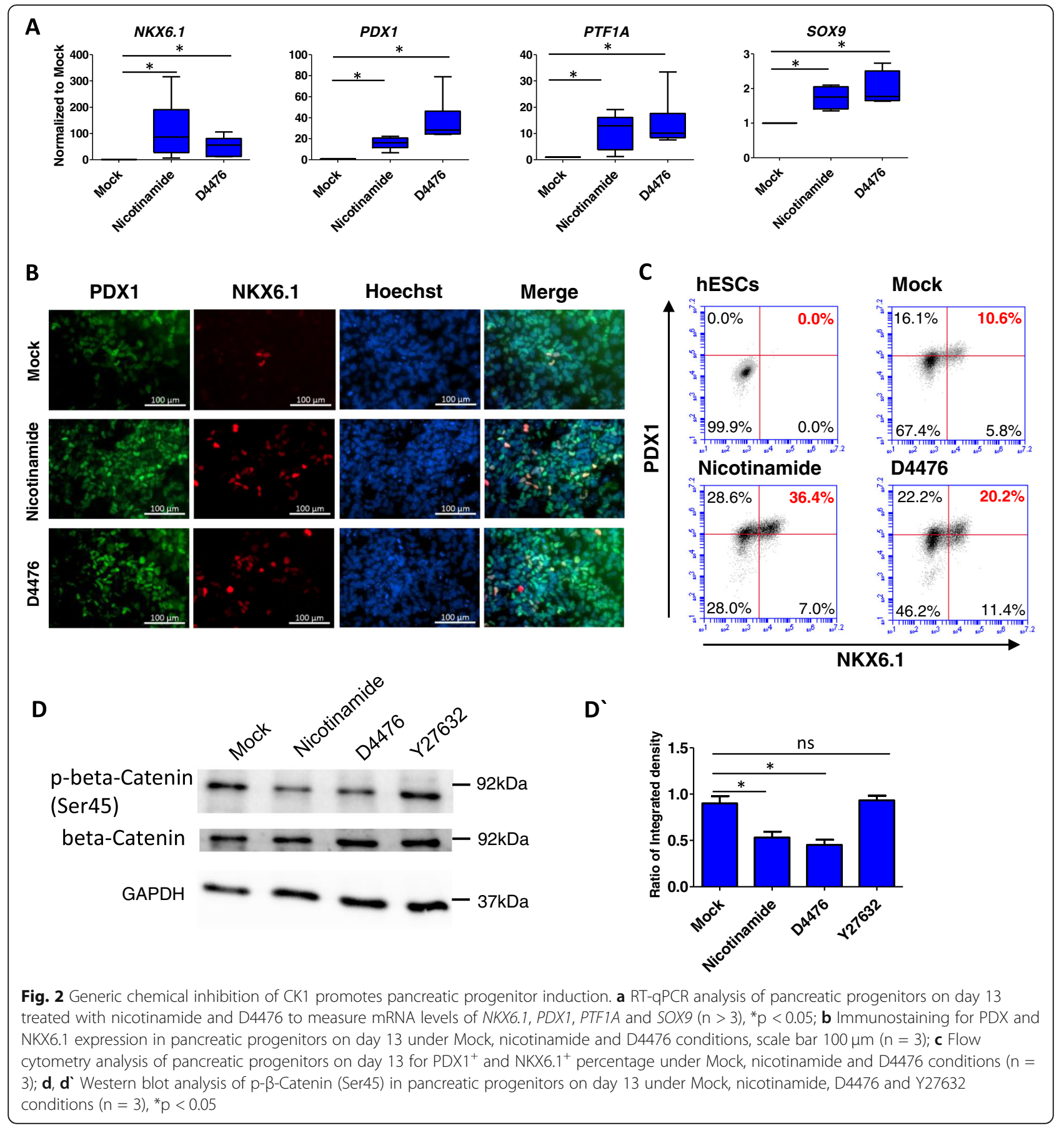

significantly decreased MLC2 phosphorylation. However, D4476 did not significantly suppress MLC2 phosphorylation (Fig. 4e, e`). These data suggest that CK1 and ROCK regulate pancreatic differentiation through distinct pathways.

We then modulated pancreatic differentiation through different combinations of ROCK and CK1 inhibition. We showed that the combination of ROCK and CK1 inhibition led to a more significant increase of $P D X 1$ and PTF1A expression, in comparison with ROCK inhibitor alone (Fig. S4b). When Y27632 or D4476 was applied to cells in the presence of nicotinamide, neither of them had an additive effect in the differentiation of $\mathrm{PDX1}^{+} / \mathrm{NKX} 6.1^{+}$cells (Fig. S4c). These results suggest that nicotinamide may induce pancreatic differentiation through a synthetic effect of CK1 and ROCK inhibition. 


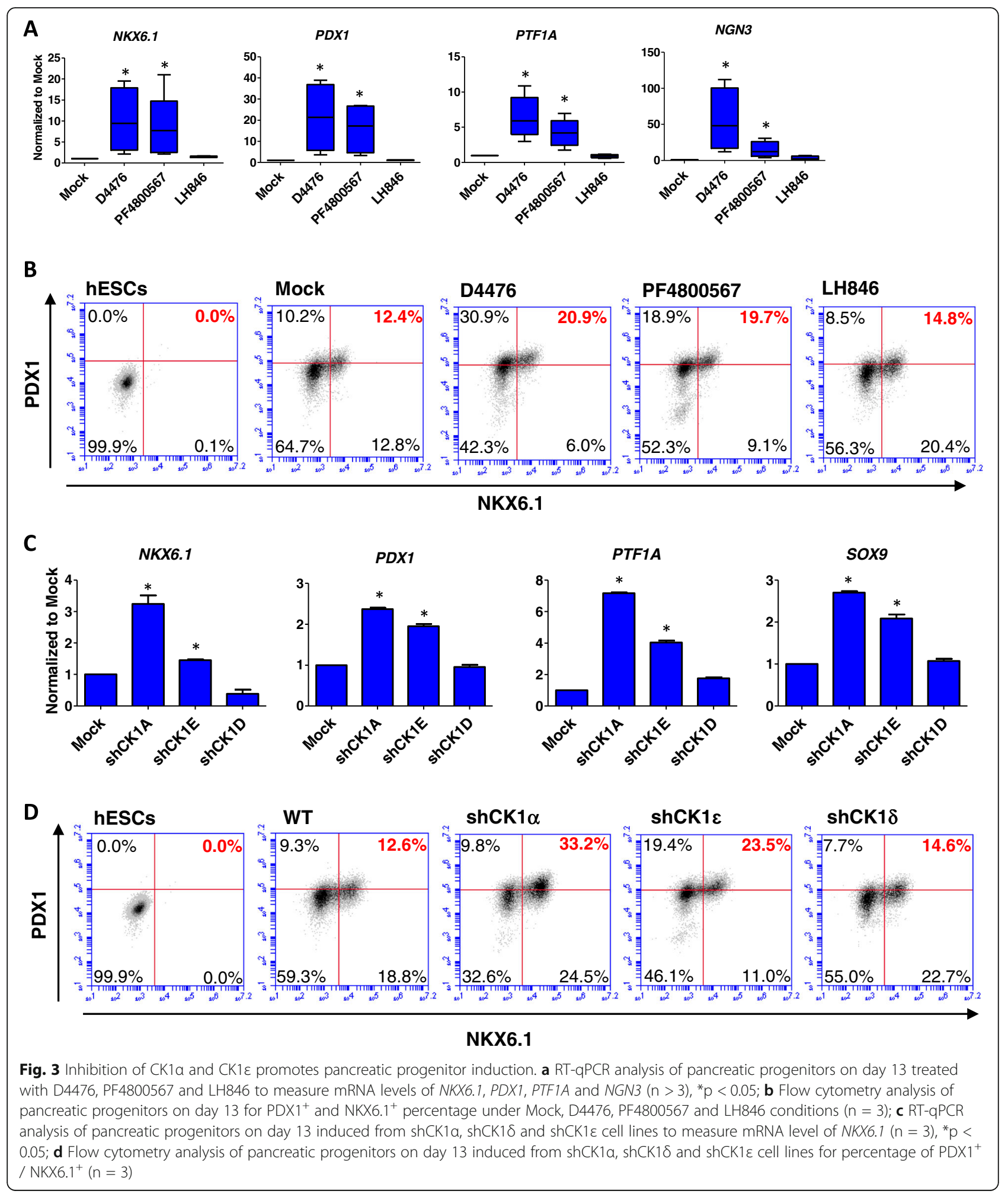




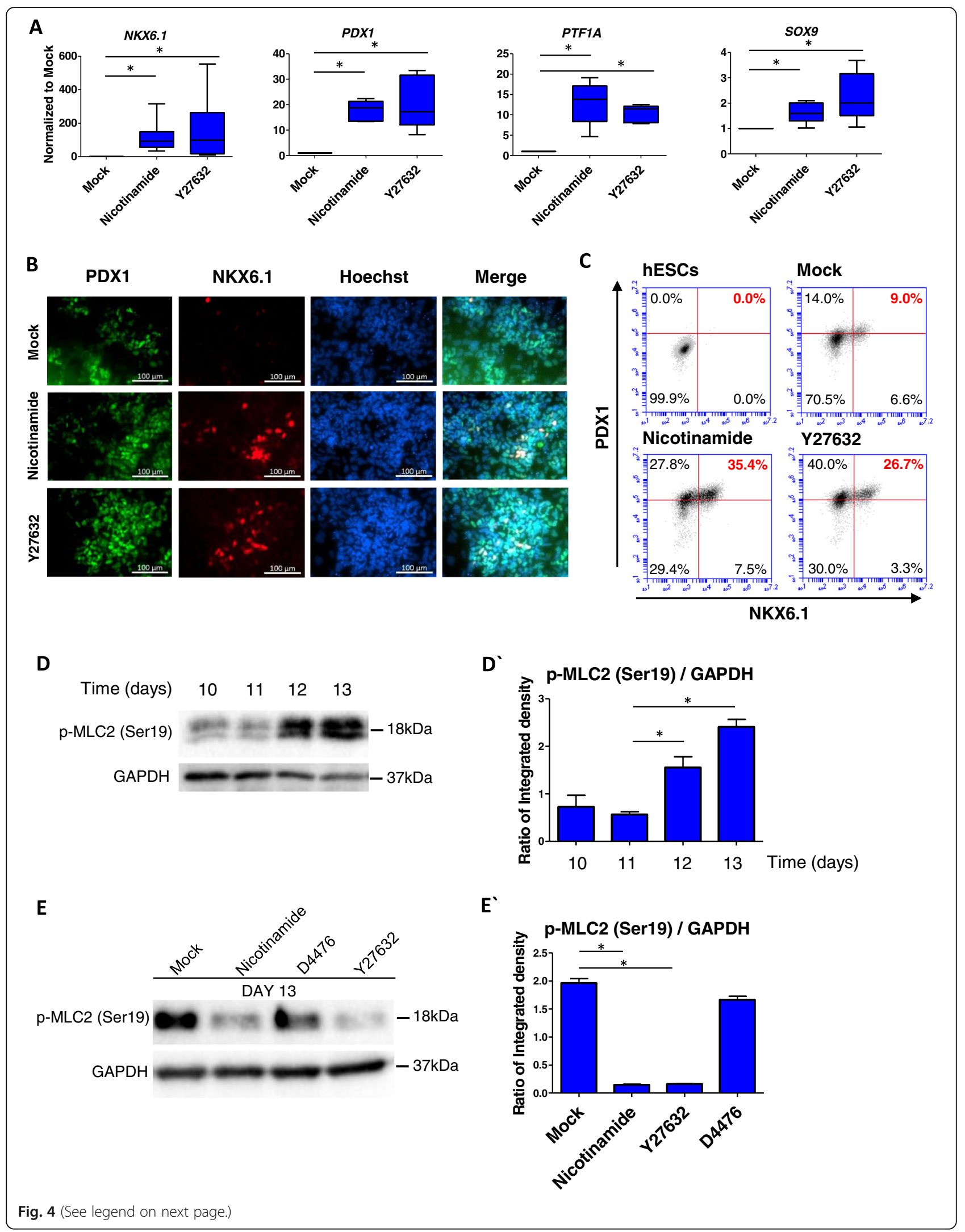


(See figure on previous page.)

Fig. 4 Nicotinamide also promotes pancreatic differentiation through ROCK inhibition. a RT-qPCR analysis of pancreatic progenitors on day 13 treated with nicotinamide and Y27632 to measure mRNA levels of NKX6.1, PDX1, PTF1A and SOX9 ( $>3$ ), ${ }^{*} p<0.05$; $\mathbf{b}$ Immunostaining for PDX and NKX6.1 expression under Mock, nicotinamide and Y27632 conditions, Scale bar $100 \mu \mathrm{m}(\mathrm{n}=3)$; c Flow cytometry analysis of pancreatic progenitors on day 13 for $\mathrm{PDX}_{1}{ }^{+}$and NKX6. $1^{+}$percentage under Mock, nicotinamide and Y27632 conditions $(n=3)$; $\mathbf{d}_{\text {, }} \mathbf{d}^{`}$ Western blot analysis of p-MLC2 (Ser19) in stage 4 (from day 10 to day 13) $(n=3)$, * $p<0.05$; e, e' Western blot analysis of p-MLC2 (Ser19) in day 13 pancreatic progenitors under Mock, nicotinamide, Y27632 and D4476 conditions $(n=3),{ }^{*} p<0.05$

\section{Global gene expression of pancreatic progenitors under different treatments}

In order to evaluate the pancreatic progenitors induced by different methods, RNA-seq profiles were obtained to examine global gene expression. In comparison to the DE condition, genes in cluster 1 (representing pancreas and stomach) were significantly upregulated by D4476, Y27632 and nicotinamide treatments, and the highly upregulated genes such as FOXA1, JAG1, ANKRD1, PFKFB3, PRSS23, CDH6, IRS1, TANC1, LAMC1, LAMC2 and CSRP1 were related to the pancreas. Meanwhile, genes in cluster 2 (representing stomach) were upregulated by D4476 or Y27632 treatment, but not by nicotinamide treatment. Genes in cluster 3 (representing pancreas) were only upregulated by D4476 treatments (Fig. 5a), and the genes of pancreatic organogenesis such as PRDM16, GATA2 and MAOA were markedly upregulated. These data indicated that specific CK1 inhibition could be a useful approach to induce a subset of pancreatic marker genes in future applications. We further showed that most pancreatic progenitor markers are upregulated in nicotinamide, CK1 inhibition and ROCK inhibition conditions (Fig. 5b). We analyzed representative gene expression in pancreatic sub-cell types by analyzing published data of a single cell analysis of the human pancreas [27]. The top 30 endocrine-specific genes were generally upregulated in each treatment. However, D4476 led to enhanced expression of more genes representing Beta cells than Y27632 and nicotinamide, indicating that the putative pancreatic progenitors generated via CK1 inhibition may be more likely to go into endocrine cell fates than ROCK inhibition and nicotinamide (Fig. 5c, Fig S5a). To further evaluate the potential of the pancreatic progenitor cells, we further induced hESCs into $\beta$-like cells (Fig. S6a), and immunostaining showed that more insulin-producing cells were generated from nicotinamide, D4476 and Y27632 treated pancreatic progenitors (Fig. S6b).

\section{Discussion}

Nicotinamide is involved in diverse biological processes, but its distinct molecular mechanisms in specific applications are not fully defined. Besides its role in metabolism and epigenetic regulation, nicotinamide is emerging as a potent modulator of kinase cascades. By examining nicotinamide function in hESC differentiation, we revealed that nicotinamide inhibits both CK1 and ROCK pathways to promote pancreatic progenitor cell fate.

Casein kinase 1 (CK1) is a family of serine/threonine kinases that are constitutively active in cells. CK1 isoforms are highly involved in circadian rhythms, nucleocytoplasmic shuttling of transcription factors, DNA transcription, and DNA repair. CK1 has been implicated in apoptosis and cell proliferation of pancreatic ductal adenocarcinoma cells, but its role in pancreatic differentiation was unknown [28]. In this study, we showed that the suppression of CK $1 \alpha$ and CK1 $1 \varepsilon$ promoted pancreatic progenitor differentiation, implying that active CK1 pathway is inhibitory to pancreatic differentiation. Nicotinamide and CK1 inhibition probably acted through WNT signaling [29] to modulate pancreatic cell fate [30]. Recent studies showed that pancreatic progenitors can be categorized into three subgroups, including $\mathrm{PDX}^{+} / \mathrm{NKX} 6.1^{+}, \quad \mathrm{PDX}^{+} / \mathrm{NKX} 6.1^{-}$, and $\mathrm{PDX}^{-} /$

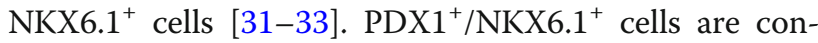
sidered as bona fide beta cell precursors, which are significantly induced by CK1 inhibitor and nicotinamide. More work is necessary to explore the differentiation of the other two subtypes in vitro.

This study also showed that nicotinamide induced pancreatic cell fate as a ROCK inhibitor. ROCK pathway is involved in glucose-stimulated insulin secretion [34] and the disassembly of glucotoxicity-induced stress fibers [35]. Recent reports showed that ROCK inhibition promoted the differentiation of $\mathrm{PDX}^{+}$posterior foregut cells into pancreatic endoderm fate at low cell density [36]. We previously showed that nicotinamide enhances hESC survival by inhibiting MLC2 phosphorylation as a ROCK inhibitor [6]. In pancreatic differentiation, nicotinamide likely promotes pancreatic progenitor fate through a similar mechanism as a ROCK inhibitor.

Although nicotinamide is best known as vitamin B3 for metabolism and inhibitors in SIRT and PARP pathways, we show that nicotinamide modulates pancreatic differentiation through its "off-target" effect as a kinase inhibitor. By exploring nicotinamide's regulation of kinase targets, 


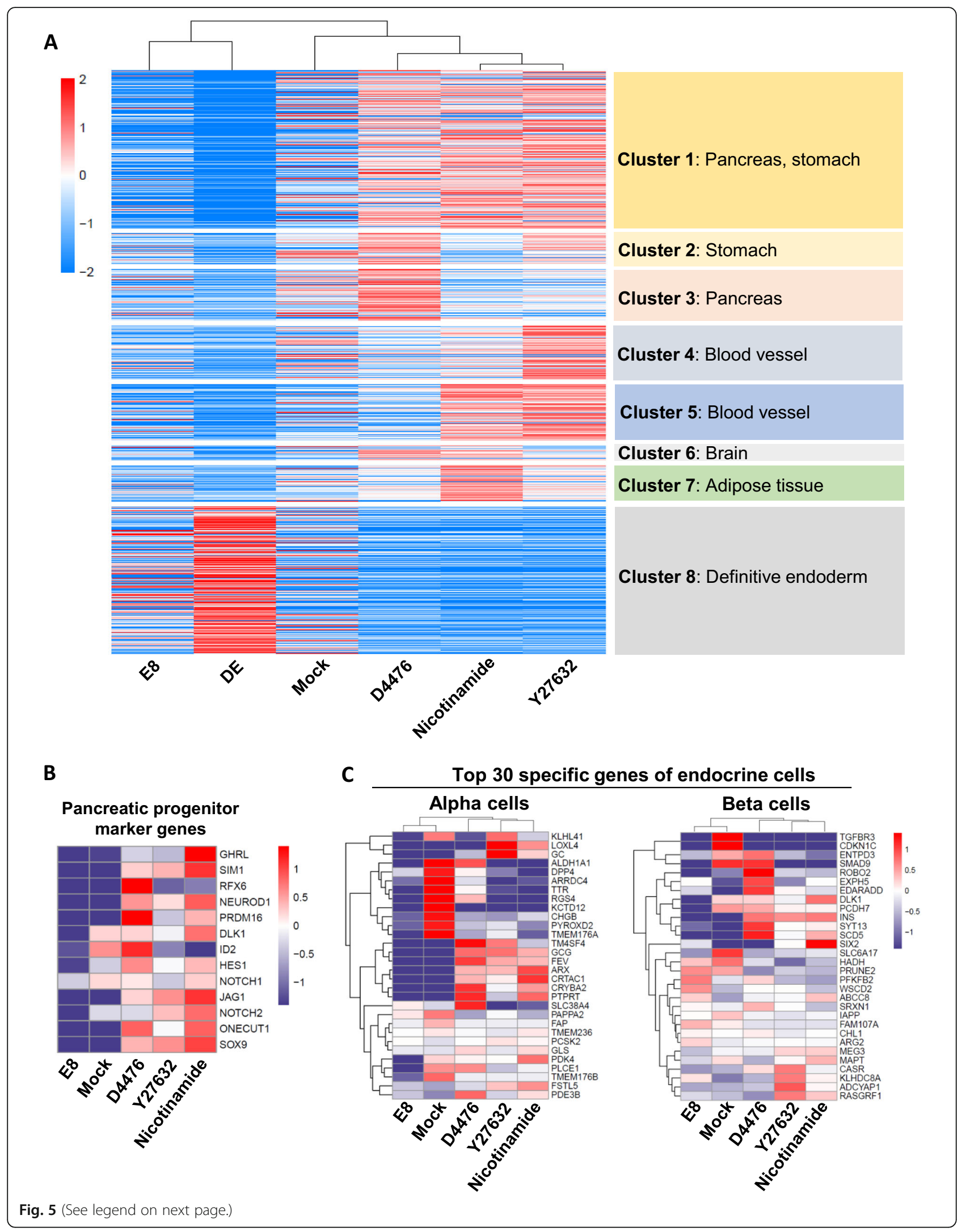


(See figure on previous page.)

Fig. 5 Global gene expression of pancreatic progenitors under different treatments. a Heatmap of genes upregulated by D4476, Y27632 and nicotinamide treatments and analysis of cell types by Enrichr. Cluster 1, 2, 3, 4, 5, 6, 7 and 8 contain 1071, 206, 344, 364, 376, 96, 243 and 999 genes, respectively; b Heatmap of representative pancreatic progenitor marker genes under Mock, D4476, Y27632, and nicotinamide conditions; $\mathbf{c}$ Heatmap and hierarchical clustering of representative genes of endocrine alpha, beta cells under Mock, D4476, Y27632 and nicotinamide conditions compared to hESCs cultured in E8

we will not only reveal the novel molecular mechanism, but also develop new methods in stem cell applications.

\section{Conclusion}

We demonstrated that the promotion of pancreatic progenitor differentiation by nicotinamide was through the dual inhibition of ROCK and CK1. These findings should be of broad interest to the stem cell community and regenerative medicine. Elucidating the mechanism of pancreatic progenitor and endocrine cell development is the foundation for in vitro induction of pancreatic progenitors and endocrine cell types.

\section{Abbreviations}

hESCs: Human embryonic stem cells; ROCK: Rho-associated protein kinase; CK1: Casein kinase; MLC2: Myosin light chain 2; NAM: Nicotinamide; NAD: Nicotinamide adenine dinucleotide; PARP: Poly (ADP-ribose) polymerases; SIRT: Sirtuins; PP: Pancreatic polypeptide cells

\section{Supplementary Information}

The online version contains supplementary material available at https://doi. org/10.1186/s13287-021-02426-2.

Additional file 1: Supplemental Figure 1. Enhancement of pancreatic progenitor induction by nicotinamide depends on kinase inhibition, but not on PARP and SIRT regulation. a Nicotinamide's molecular targets screened via KINOMEscan ${ }^{\mathrm{TM}}$ confirmed that nicotinamide can directly bind and inhibit CK1 and ROCK; b RT-qPCR analysis for mRNA levels of NKX6.1 and PDX1 in pancreatic progenitors on day 13 of differentiation from $\mathrm{H9}$, treated with nicotinamide, D4476 and Y27632 ( $n=3),{ }^{*} p<0.05$; C RT-qPCR analysis of pancreatic progenitors on day 13 for mRNA levels of NKX6.1 and PDX1 under Mock, nicotinamide (10 mM), niacin (1, 5, 10 mM), PARP inhibitor ABT888 (10, 50, $500 \mathrm{nM})$ and SIRT inhibitor EX527 (1, $10,20 \mu M)$ conditions $(n=3)$; $d$ Flow cytometry analysis to test the effect of nicotinamide (10 mM), niacin (5 mM), ABT888 (50 nM) and EX527 $(10 \mu \mathrm{M})$ on generation of PDX $1^{+} / \mathrm{NKX} 6.1^{+}$cells $(\mathrm{n}=3)$; e Flow cytometry analysis to test the effect of nicotinamide $(10 \mathrm{mM})$, D4476 $(5 \mu \mathrm{M})$ and Y27632 $(10 \mu M)$ on the generation of PDX $1^{+} / \mathrm{NKX} 6.1^{+}$cells $(n=3)$. Supplemental Figure 2.Generic chemical inhibition of CK1 promotes pancreatic progenitor induction. a RT-qPCR analysis of pancreatic progenitors on day 13 treated with nicotinamide and D4476 to measure mRNA levels of NEUROD1 and NGN3 ( $n>3)$, * $p<0.05$. Supplemental Figure 3. Inhibition of CK1 $a$ and $C K 1 \varepsilon$ promotes pancreatic progenitor induction. a RTqPCR analysis to measure mRNA levels of $C K 1 a, C K 1 \varepsilon$ and $C K 1 \delta$ in shCK1a, shCK $1 \varepsilon$ and shCK1 $\delta$ cell lines in pluripotency stage $(n=3),{ }^{*} p<0.05 ; b$ Western blot analysis to assay protein level of CK1a, CK1 $\varepsilon$ and $\mathrm{CK} 1 \delta$ in shCK1a, shCK1 1 and shCK1 $\delta$ cell lines in pluripotency stage; c Flow cytometry analysis of pancreatic progenitors on day 13 induced from shCK1a, shCK1 $\delta$ and shCK1 1 cell lines for percentage of PDX $1^{+} / \mathrm{NKX} 6.1^{+}$ $(n=3)$. Supplemental Figure 4. Nicotinamide also promotes pancreatic differentiation through ROCK inhibition. a RT-qPCR analysis of pancreatic progenitors on day 13 treated with nicotinamide and Y27632 to measure mRNA levels of NEUROD1 and NGN3 ( $n>3)$, * $p<0.05$; b RT-qPCR analysis of pancreatic progenitors on day 13 treated with nicotinamide, Y27632, D4476 and Y27632+D4476 to measure mRNA levels of NKX6.1, PDX1 and PTF1A $(n>3)$, * $p<0.05$; c Flow cytometry analysis to test the effect of nicotinamide, nicotinamide+D4476 and nicotinamide+Y27632 on the generation of $\mathrm{PDX} 1^{+} / \mathrm{NKX} 6.1^{+}$cells $(n=3)$. Supplemental Figure 5. Global gene expression of pancreatic progenitors under different treatments. a Heatmap and hierarchical clustering of representative genes of delta, epsilon, pancreatic polypeptide (PP), Acinar and ductal cells under Mock, D4476, Y27632 and nicotinamide conditions compared to hESCs cultured in E8. Supplemental Figure 6. Pancreatic progenitors under Mock, nicotinamide, D4476 and Y27632 treatment can be further induced into insulin positive $\beta$-like cells. a Differentiation strategy from pancreatic progenitors to $\beta$-like cells; $b$ Immunostaining for insulin expression in $\beta$ like cells generated from pancreatic progenitors under Mock, nicotinamide, D4476 and Y27632 condition, Scale bar $400 \mu \mathrm{M}$ and $100 \mu \mathrm{m}$.

Additional file 2. Primers used in RT-qPCR analysis.

Additional file 3. Antibodies used in flow cytometry, immunostaining or Western Blot analysis.

Additional file 4. Chemicals or recombinant proteins used in hESC differentiation.

\section{Acknowledgements}

Dr. Ruiyu Xie and Dr. Xinwei Wu of the University of Macau provided valuable suggestions to this project. We want to thank the council members of the Macau Society for Stem Cell Research (MSSCR) for the constructive discussions.

\section{Authors' contributions}

YZ, YM and WL conducted stem cell maintenance and differentiation; YZ, YM and $L L$ analyzed nicotinamide function; $Y Z$ characterized the differentiated cells; YZ, JX, ZR and ZZ conducted bioinformatics analysis; $Y Z$ and GC designed the experiments and analyzed data; $Y Z, W L$ and $G C$ wrote the manuscript. All authors read and approved the final manuscript.

\section{Funding}

This project was funded by the University of Macau (File No. MYRG201800135-FHS and MYRG2019-00147-FHS), and also by the Science and Technology Development Fund, Macau SAR (File No. 131/2014/A3, 056/2015/A2, 0059/2019/A1, 0123/2019/A3 and FDCT/0011/2019/AKP).

Availability of data and materials

The data supporting the finding of this article are all online.

\section{Declarations}

\section{Ethics approval and consent to participate}

The use of hESCs was approved by the Institutional Review Board at the University of Macau.

\section{Competing interests}

The authors declare that they have no competing interests.

\section{Author details}

${ }^{1}$ Centre of Reproduction, Development and Aging, Faculty of Health Sciences, University of Macau, Macau SAR, China. ${ }^{2}$ Institute of Translational Medicine, Faculty of Health Sciences, University of Macau, Macau SAR, China. ${ }^{3}$ Zhuhai Precision Medical Center, Zhuhai People's Hospital, Jinan University, Zhuhai, Guangdong, China. ${ }^{4}$ Bioimaging and Stem Cell Core Facility, Faculty of Health Sciences, University of Macau, Macau SAR, China. ${ }^{5}$ State Key Laboratory of Cardiovascular Disease, Beijing Key Laboratory for Molecular Diagnostics of Cardiovascular Diseases, Diagnostic Laboratory Service, Fuwai Hospital, National Center for Cardiovascular Diseases, Chinese Academy of Medical Sciences and Peking Union Medical College, Beijing, China. ${ }^{6} \mathrm{MoE}$ 
Frontiers Science Center for Precision Oncology, University of Macau, Macau SAR, China.

\section{Received: 1 March 2021 Accepted: 28 May 2021}

Published online: 25 June 2021

\section{References}

1. Wang Y, Tang Z, Gu P. Stem/progenitor cell-based transplantation for retinal degeneration: a review of clinical trials. Cell Death Dis. 2020;11(9):1-4.

2. Ahmed RE, Anzai T, Chanthra N, Uosaki H. A brief review of current maturation methods for human induced pluripotent stem cells-derived cardiomyocytes. Front Cell Dev Biol. 2020;19(8):178.

3. Salehi-Pourmehr H, Hajebrahimi S, Rahbarghazi R, Pashazadeh F, Mahmoudi J, Maasoumi $\mathrm{N}$, et al. Stem cell therapy for neurogenic bladder dysfunction in rodent models: a systematic review. Int Neurourol J. 2020;24(3):241-57. https://doi.org/10.5213/inj.2040058.029.

4. Koster C, Wever KE, Wagstaff PE, Hirk KT, Hooijmans CR, Bergen AA. A Systematic Review on Transplantation Studies of the Retinal Pigment Epithelium in Animal Models. Int J Mol Sci. 2020;21(8):2719. https://doi.org/1 0.3390/ijms21082719.

5. Okabe K, et al. Implications of altered NAD metabolism in metabolic disorders. J Biom Sci. 2019;26(1):34.

6. Meng Y, Ren Z, Xu F, Zhou X, Song C, Wang VY, et al. Nicotinamide promotes cell survival and differentiation as kinase inhibitor in human pluripotent stem cells. Stem Cell Rep. 2018;11(6):1347-56. https://doi.org/1 0.1016/.jstemcr.2018.10.023

7. Ungerstedt JS, Blombäck M, Söderström T. Nicotinamide is a potent inhibitor of proinflammatory cytokines. Clin Exp Immunol. 2003;131(1):4852. https://doi.org/10.1046/j.1365-2249.2003.02031.x.

8. Hwang ES, Song SB. Nicotinamide is an inhibitor of SIRT1 in vitro, but can be a stimulator in cells. Cell Mol Life Sci. 2017;74(18):3347-62. https://doi. org/10.1007/s00018-017-2527-8.

9. Patwa J, Khan S, Jena G. Nicotinamide attenuates cyclophosphamide induced hepatotoxicity in SD rats by reducing oxidative stress and apoptosis. J Biochem Mol Toxicol. 2020;34(10):e22558.

10. Kim KP, Choi J, Yoon J, Bruder JM, Shin B, Kim J, et al. Permissive epigenomes endow reprogramming competence to transcriptional regulators. Nat Chem Biol. 2020;17:1-10

11. Peled T, Shoham H, Aschengrau D, Yackoubov D, Frei G, Lerrer B, et al. Nicotinamide, a SIRT1 inhibitor, inhibits differentiation and facilitates expansion of hematopoietic progenitor cells with enhanced bone marrow homing and engraftment. Exp Hematol. 2012;40(4):342-55.

12. Buchholz DE, Pennington BO, Croze RH, Hinman CR, Coffey PJ, Clegg DO. Rapid and efficient directed differentiation of human pluripotent stem cells into retinal pigmented epithelium. Stem Cells Transl Med. 2013;2(5):384-93. https://doi.org/10.5966/sctm.2012-0163.

13. Parsons XH, Teng YD, Parsons JF, Snyder EY, Smotrich DB, Moore DA. Efficient derivation of human cardiac precursors and cardiomyocytes from pluripotent human embryonic stem cells with small molecule induction. JOVE. 2011;3(57):e3274.

14. Rezania A, Bruin JE, Arora P, Rubin A, Batushansky I, Asadi A, et al. Reversal of diabetes with insulin-producing cells derived in vitro from human pluripotent stem cells. Nat Biotechnol. 2014;32(11):1121-33. https://doi.org/1 $0.1038 /$ nbt.3033.

15. Lynn FC, Smith SB, Wilson ME, Yang KY, Nekrep N, German MS. Sox9 coordinates a transcriptional network in pancreatic progenitor cells. Proc Natl Acad Sci. 2007;104(25):10500-5. https://doi.org/10.1073/pnas. 0704054104

16. Petersen MB, Azad A, Ingvorsen C, Hess K, Hansson M, Grapin-Botton A, et al. Single-cell gene expression analysis of a human ESC model of pancreatic endocrine development reveals different paths to $\beta$-cell differentiation. Stem Cell Rep. 2017;9(4):1246-61. https://doi.org/10.1016/j. stemcr.2017.08.009.

17. Russ HA, Parent AV, Ringler JJ, Hennings TG, Nair GG, Shveygert M, et al. Controlled induction of human pancreatic progenitors produces functional beta-like cells in vitro. EMBO J. 2015;34(13):1759-72. https://doi.org/10.152 52/embj.201591058.

18. Pagliuca FW, Millman JR, Gürtler M, Segel M, Van Dervort A, Ryu JH, et al. Generation of functional human pancreatic $\beta$ cells in vitro. Cell. 2014;159(2): 428-39. https://doi.org/10.1016/j.cell.2014.09.040.
19. Ghazizadeh Z, Kao DI, Amin S, Cook B, Rao S, Zhou T, et al. ROCKIl inhibition promotes the maturation of human pancreatic beta-like cells. Nat Commun. 2017;8(1):1-2.

20. Wedeken L, Luo A, Tremblay JR, Rawson J, Jin L, Gao D, et al. Adult murine pancreatic progenitors require epidermal growth factor and nicotinamide for self-renewal and differentiation in a serum-and conditioned mediumfree culture. Stem Cells Dev. 2017;26(8):599-607. https://doi.org/10.1089/ scd.2016.0328.

21. Cho YM, Lim JM, Yoo DH, Kim JH, Chung SS, Park SG, et al. Betacellulin and nicotinamide sustain PDX1 expression and induce pancreatic $\beta$-cell differentiation in human embryonic stem cells. Biochem Biophys Res Commun. 2008;366(1):129-34. https://doi.org/10.1016/j.bbrc.2007.11.112.

22. Nostro MC, Sarangi F, Yang C, Holland A, Elefanty AG, Stanley EG, et al. Efficient generation of NKX6-1+ pancreatic progenitors from multiple human pluripotent stem cell lines. Stem Cell Rep. 2015;4(4):591-604. https:// doi.org/10.1016/j.stemcr.2015.02.017.

23. Lin $Y$, Chen G. Embryoid body formation from human pluripotent stem cells in chemically defined E8 media. StemBook. Cambridge: Harvard Stem Cell Institute; 2014.

24. Beers J, Gulbranson DR, George N, Siniscalchi LI, Jones J, Thomson JA, et al. Passaging and colony expansion of human pluripotent stem cells by enzyme-free dissociation in chemically defined culture conditions. Nat Protoc. 2012;7(11):2029-40. https://doi.org/10.1038/nprot.2012.130.

25. Hagen T, Vidal-Puig A. Characterisation of the phosphorylation of $\beta$-catenin at the GSK-3 priming site Ser45. Biochem Biophys Res Commun. 2002; 294(2):324-8. https://doi.org/10.1016/S0006-291X(02)00485-0.

26. Chen G, Hou Z, Gulbranson DR, Thomson JA. Actin-myosin contractility is responsible for the reduced viability of dissociated human embryonic stem cells. Cell Stem Cell. 2010;7(2):240-8. https://doi.org/10.1016/j. stem.2010.06.017.

27. Muraro MJ, Dharmadhikari G, Grün D, Groen N, Dielen T, Jansen E, et al. A single-cell transcriptome atlas of the human pancreas. Cell Syst. 2016;3(4): 385-94. https://doi.org/10.1016/j.cels.2016.09.002.

28. Vena F, Bayle S, Nieto A, Quereda V, Aceti M, Frydman SM, et al. Targeting casein kinase 1 delta sensitizes pancreatic and bladder cancer cells to gemcitabine treatment by upregulating deoxycytidine kinase. Mol Cancer Ther. 2020;19(8):1623-35. https://doi.org/10.1158/1535-7163.MCT-19-0997.

29. Cruciat C-M. Casein kinase 1 and Wnt/3-catenin signaling. Curr Opin Cell Biol. 2014;31:46-55. https://doi.org/10.1016/j.ceb.2014.08.003.

30. Sharon N, Vanderhooft J, Straubhaar J, et al. Wnt signaling separates the progenitor and endocrine compartments during pancreas development. Cell Rep. 2019;27(8):2281-2291. e5.

31. Rezania A, Bruin JE, Xu J, Narayan K, Fox JK, O'Neil JJ, et al. Enrichment of human embryonic stem cell-derived NKX6.1-expressing pancreatic progenitor cells accelerates the maturation of insulin-secreting cells in vivo. Stem Cells. 2013;31(11):2432-42. https://doi.org/10.1002/stem.1489.

32. Aigha II, Memon B, Elsayed AK, Abdelalim EM. Differentiation of human pluripotent stem cells into two distinct NKX6.1 populations of pancreatic progenitors. Stem Cell Res Ther. 2018;9(1):1-1.

33. Memon B, Younis I, Abubaker F, Abdelalim EM. PDX1-/NKX6.1+ progenitors derived from human pluripotent stem cells as a novel source of insulinsecreting cells. Diab Metab Res Rev. 2020. p. e3400. https://onlinelibrary. wiley.com/doi/full/10.1002/dmrr.3400.

34. Hammar E, Tomas A, Bosco D, Halban PA. Role of the Rho-ROCK (Rhoassociated kinase) signaling pathway in the regulation of pancreatic $\beta$-cell function. Endocrinology. 2009;150(5):2072-9. https://doi.org/10.1210/en.2 008-1135.

35. Kong X, Yan D, Sun J, Wu X, Mulder H, Hua X, et al. Glucagon-like peptide 1 stimulates insulin secretion via inhibiting RhoA/ROCK signaling and disassembling glucotoxicity-induced stress fibers. Endocrinology. 2014 155(12):4676-85. https://doi.org/10.1210/en.2014-1314.

36. Toyoda T, Kimura A, Tanaka H, Ameku T, Mima A, Hirose Y, et al. Rhoassociated kinases and non-muscle myosin Ils inhibit the differentiation of human iPSCs to pancreatic endoderm. Stem Cell Rep. 2017;9(2):419-28. https://doi.org/10.1016/j.stemcr.2017.07.005.

\section{Publisher's Note}

Springer Nature remains neutral with regard to jurisdictional claims in published maps and institutional affiliations. 